\title{
True Random Number Generator using Fish Tank Image
}

\author{
Rajat Katyal \\ University of Pune \\ S.C.O.E. - Dept. of I.T. \\ Vadgaon (Bk), Pune-41, India
}

\author{
Ankit Mishra \\ University of Pune \\ S.K.N.C.O.E. - Dept. of I.T. \\ Vadgaon (Bk), Pune-41, India
}

\author{
Adarsh Baluni \\ University of Pune \\ S.K.N.C.O.E. - Dept. of I.T. \\ Vadgaon (Bk), Pune-41, India
}

\begin{abstract}
A Pseudo Random Number Generator (PRNG) uses a deterministic system and an initial seed to generate random numbers. In order for the output sequence to be truly random, a truly random input seed is used. Most True Random Number Generators (TRNG), use noise in the form nuclear decay, atmospheric noise, electrical noise or Brownian motion as their initial seed.

In order to reduce the computational complexity, we use a simple setup of a fish tank as the variable environment, capturing its images over time. The image data is then applied to a reduction algorithm and hash function to generate the initial seed. We propose a cost efficient method of extracting the true seed from the image data and applying it to a pseudo random generator, a Linear Congruential Generator (LCG) in our case to give true random numbers.
\end{abstract}

\section{General Terms}

PRNG, TRNG, LCG

\section{Keywords}

True random number generator, Image data

\section{INTRODUCTION}

Random numbers are the numbers that are part of a sequence in which values are uniformly distributed over a definite set and any future value cannot be reliably predicted based on the existing set of values. The sequence is produced by what is usually called as a random number generator. There are two types of random number generators: pseudo - random number generator and true random number generator. A pseudo random number generator uses a deterministic algorithm which produces random numbers based on some initial value known as seed. Most random number sources actually utilize a pseudo-random generator. Since the output is purely a function of the seed data, the actual entropy of the output can never exceed the entropy of the seed [2].In certain real world secure systems the quality of random numbers is of paramount importance. For such applications it is not recommendable to use PRNGs as they use a deterministic algorithm. The output for such PRNGs are same for a particular seed, hence it is obvious that they cannot be secure if the seed is predictable in any which way.

TRNGs however use physical phenomena that are unpredictable in nature to generate true random numbers. The physical phenomena that are currently used are radioactive decay, atmospheric noise, nuclear decay, Brownian motion, clock drifts etc [9]. These results are then applied to cryptographic hash function for obtaining uniformly distributed outputs. Unfortunately one does not find these methods as peripherals for the modern day PC's making them very difficult to implement.

We have used a simple fish-tank setup as the variable environment and capturing its images over time as the initial seed. A different image is used every time to generate a set of random numbers. The image data provides the initial seed which is extracted using a reduce algorithm and hashing. This truly random seed is then fed to a pseudo random algorithm, a linear congruential generator in our case, to give a set of random numbers.

\section{NEED}

Random numbers are required in a lot of areas. Games, statistical sampling, Monte Carlo method simulation, gambling and lottery, sort and hash algorithms are a few of the fields that utilize the element of randomness.

The extensive utility of random numbers lies in the real world security systems for exchange of classified data. Cryptography uses random numbers to a great extent for example while generating encryption key pairs and digital signatures requiring a high degree of randomness [10].

\section{GENERATION}

\subsection{Existing Methodologies}

The Hardware random number generators use different sources of entropy to calculate the initial seed values. These generally require complex hardware setups. Some of the commonly used methods are:

- Radioactive material decay time

- Atmospheric Noise

- Electrical noise from resistors or semiconductors

A similar approach to ours is to use a randomness extractor such as cryptographic hash function against certain nonuniformly random source.

This technique had been used by the Lavarnd generator that took images of the patterns of floating point materials in a lava lamp, extracting the seed and feeding it to a pseudo random number generator.

\subsection{Our Approach}

We have used a fish tank setup having over 20 live fishes as our variable environment source. Clicking its images over time we get the random and unpredictable image data. The image data is then applied to a reduce algorithm and hashing to generate a true and unpredictable seed. 
This seed is fed to a pseudo random algorithm similar to that of a linear congruential algorithm in order to generate truly random numbers [14].

We have used the GIF image format having $640 \times 480$ pixels, in order to reduce the computational time and complexity. Two image samples are shown below along with their true seed values.

\subsection{Samples}

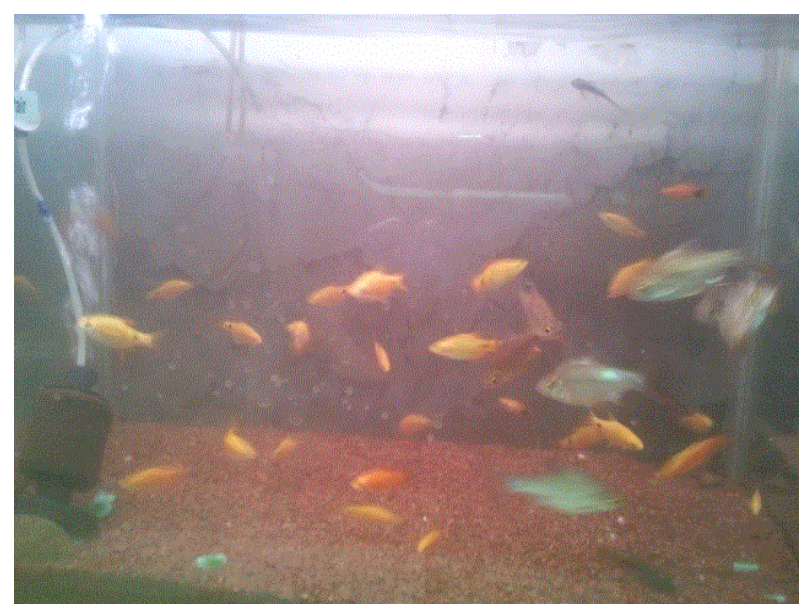

Fig 1: Sample Image A

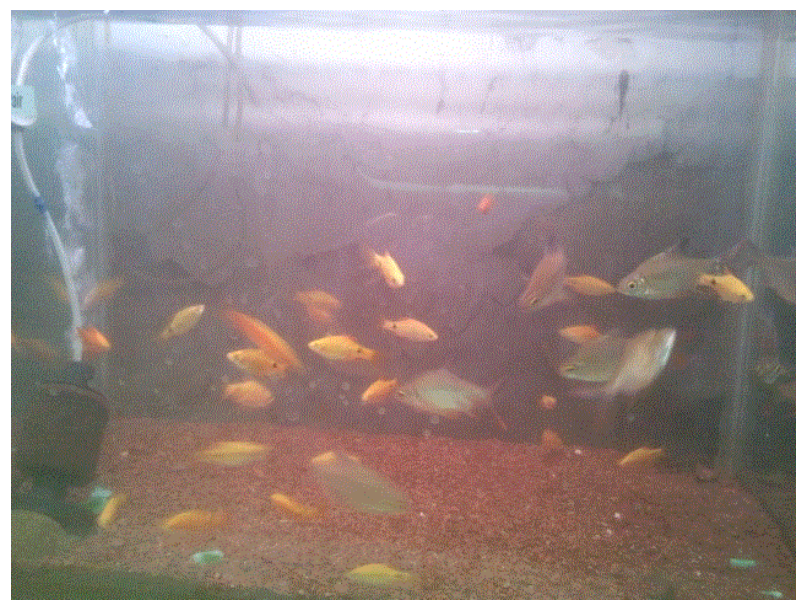

Fig 2: Image Sample B

In order to generate the different set of random numbers, we use a different image samples. For the shown image samples, the generated true initial seeds were:

\section{Image A: 16338}

Image B: 3453

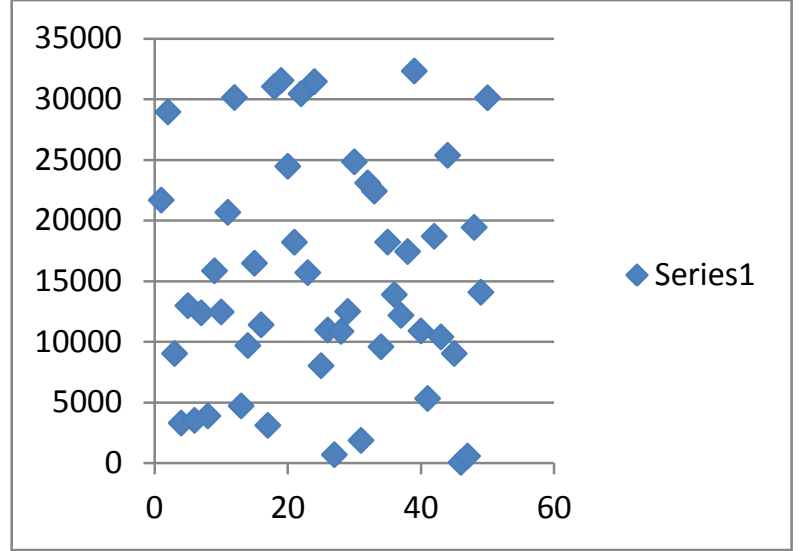

Fig 3: Sample set of 50 possitive random numbers

A small sample set of 50 random numbers produced using our generator is shown in Fig 3. The sample has a range of 0 32768. The figure illustrates the randomness of the generated numbers.

\subsection{FLOWCHART}

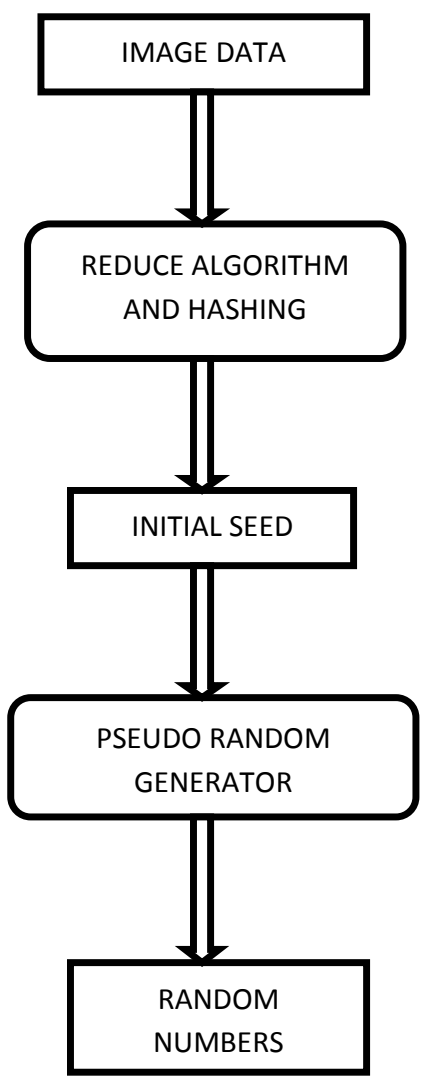

Fig 4: Flowchart of True Random Number Generator

\section{TESTING}

There are a wide variety of testing methods available that are carried out to check the quality of generated random numbers. These are typically applied to a PRNG for its quality check or any new type of random number generator [11].

Since we utilize an already proven pseudo random algorithm, a linear congruential generator, we did a quality test for our chosen variables and the initial seed. The observed pattern showed a high quality of randomness. 
A single type of test cannot determine the quality of randomness. Also in a few tests, a vague pattern of result can actually mean higher quality of randomness as it is basically a factor of unpredictability.

A Runs Test carried out on 80 numbers (below Table 1) yields the following result. Such a test alone cannot be a quality testing factor for randomness, but is one of the essential tests.

Some other known testing methods are are frequency test, serial test, poker test and gap test.

Table 1: Runs Test carried on a sample of 80 numbers

\begin{tabular}{|c|c|c|c|c|}
\hline 7053 & 7716 & 1270 & 6327 & 3041 \\
\hline 5846 & 3780 & 3705 & 444 & 6035 \\
\hline 4682 & 6603 & 5612 & 8623 & 893 \\
\hline 4319 & 8631 & 4384 & 980 & 4195 \\
\hline 1618 & 5393 & 3641 & 7440 & 7430 \\
\hline 7954 & 2538 & 5232 & 4324 & 5356 \\
\hline 1919 & 320 & 8676 & 1102 & 2864 \\
\hline 4830 & 5704 & 749 & 1773 & 3347 \\
\hline 7545 & 8503 & 914 & 3770 & 1524 \\
\hline 2219 & 4633 & 3774 & 7635 & 1939 \\
\hline 63 & 2391 & 5476 & 1662 & 2766 \\
\hline 2541 & 3267 & 7942 & 1667 & 8222 \\
\hline 5249 & 1920 & 8396 & 1151 & 5693 \\
\hline 1683 & 6473 & 3452 & 270 & 6502 \\
\hline 1246 & 4585 & 290 & 2133 & 8577 \\
\hline 7928 & 2106 & 69 & 7192 & 3981 \\
\hline \multicolumn{3}{|c|}{ Sample Set } & \multicolumn{2}{|c|}{80} \\
\hline \multicolumn{3}{|c|}{ Number of Runs(R) } & \multicolumn{2}{|c|}{50} \\
\hline \multicolumn{3}{|c|}{ P-Value } & \multicolumn{2}{|c|}{0.02107} \\
\hline \multicolumn{3}{|c|}{ Conclusion } & \multicolumn{2}{|c|}{$\begin{array}{l}\text { Moderate evidence } \\
\text { against Sequential } \\
\text { Randomness }\end{array}$} \\
\hline
\end{tabular}

\section{RESULT AND CONCLUSION}

The random number generator is truly random as the seed value used is taken from an unpredictable source. Hence it completely avoids the possibility of determinism which is a risk factor in pseudo random number generators.

The generator yields truly random numbers up to 16 bits or 65536 with a high degree of randomness. The random number generation can be divided mainly into two phases: Image to seed conversion and Pseudo random generation.

It is also much easier to implement as compared to other hardware random number generators which use radioactive decay, atmospheric noise, electrical noise, etc. and require a much complex hardware setup to generate randomness. The TRNG using fish tank image data is a cost efficient alternative to generate truly random numbers.

\section{LIMITATIONS AND FUTURE SCOPE}

\section{Image processing time period}

Although the pseudo random processing time is comparable to any good PRNG but it involves an additional overhead of image processing time which makes it slightly slower.

The Future Scope of the True Random Number Generator is to reduce the overall processing time. This can be done by using a camera to generate the hexadecimal image data and using it directly, thereby reduce the GIF image conversion overhead.

\section{Output range: $16 \mathrm{bit}$}

The 16bit range (0-65536) of random numbers can be increased by using a bigger sized compiler and microprocessor, while maintaining the high degree of randomness.

\section{ACKNOWLEDGMENTS}

Our thanks to P.Hellekalek and everybody that has contributed in generating good quality random numbers. Also special thanks to our friend Tejaswi Bhutada.

\section{REFERENCES}

[1] P. Hellekalek, Good random number generators are (not so) easy to find, Mathematics and Computers in Simulation 46 (1998) 485-505.

[2] Benjamin Jun and Paul Kocher, P. 1999 The Intel Random Number Generator. Technical Report. University of Maryland at College Park.

[3] N. K Pareek, V. Patidar, K. K Sud, A Rndom Bit Generator Using Chaotic Maps, International Journal of Network Security, Vol. 10, no. 1, pp. 32-38, 2010.

[4] Tang, H.C., "An Analysis of Linear Congruential Random Number Generators when Multiplier Restrictions Exist," European Journal of Operational Research, Vol. 182, pp. 820828 (2007)

[5] Hedayatpour, S., Chuprat, Suriayati, Hash functionsbased random number generator with image data source Open Systems (ICOS), 2011 IEEE.

[6] Xuan Li, Guoji Zhang, Yuliang Liao, Chaos-based true random number generator using image

[7] P. Murali and R.Palraj, True Random Number Generator Based on Image for Key Exchange program.

[8] Xuan Li; Guoji Zhang; Yuliang Liao, "Chaos-based true random number generator using image," Computer Science and Service System (CSSS), 2011 International Conference on , vol., no., pp.2145,2147, 27-29 June 2011 doi: 10.1109/CSSS.2011.5974933

[9] Matsumoto, M. and T. Nishimura. "Mersenne Twister: A 623-Dimensionally Equidistributed Uniform Pseudorandom Number Generator." ACM Transactions on Modeling and Computer Simulation 8, no. 1 (1998): $3 \mid 30$.

[10] Volos, C.K.; Kyprianidis, I. M.; Stouboulos, I. N., "Image encryption process based on a chaotic True Random Bit Generator," Digital Signal Processing, 2009 16th International Conference on, vol., no., pp.1,4, 5-7 July 2009 doi: 10.1109/ICDSP.2009.5201107

[11] Gentle, J. E. Random Number Generation and Monte Carlo Methods, (2nd Ed.) SpringerVerlag, 2003.

[12] Geman, S. and D. Geman. "Stochastic Relaxation, Gibbs Distributions, and the Bayesian

[13] Restoration of Images." IEEE Transactions on Pattern Analysis and Machine Intelligence 6, no. 6 (1984): $721 \mid 741$

[14] Junod, P. "Cryptographic Secure Pseudo-Random Bits Generation: The Blum|Blum|Shub Generator." Augus 1999.http://crypto.junod.info/bbs.pdf 